\title{
Pairing Interactions and Gibbs Adsorption at the Liquid Bi-In Surface: A Resonant X-Ray Reflectivity Study
}

\author{
E. DiMasi, ${ }^{1, *}$ H. Tostmann, ${ }^{2, \dagger}$ O. G. Shpyrko, ${ }^{2}$ P. Huber, ${ }^{2}$ B. M. Ocko, ${ }^{1}$ P. S. Pershan, ${ }^{2}$ M. Deutsch, ${ }^{3}$ and L. E. Berman ${ }^{4}$ \\ ${ }^{1}$ Department of Physics, Brookhaven National Laboratory, Upton, New York 11973 \\ ${ }^{2}$ Division of Engineering and Applied Sciences and Department of Physics, Harvard University, Cambridge, Massachusetts 02138 \\ ${ }^{3}$ Department of Physics, Bar-Ilan University, Ramat-Gan 52900, Israel \\ ${ }^{4}$ National Synchrotron Light Source, Brookhaven National Laboratory, Upton, New York 11973
}

(Received 2 August 2000)

\begin{abstract}
Resonant X-ray reflectivity measurements from the surface of liquid $\mathrm{Bi}_{22} \mathrm{In}_{78}$ find only a modest surface $\mathrm{Bi}$ enhancement, with 35 at. \% $\mathrm{Bi}$ in the first atomic layer. This is in contrast to the Gibbs adsorption in all liquid alloys studied to date, which show surface segregation of a complete monolayer of the low surface tension component. This suggests that surface adsorption in Bi-In is dominated by attractive interactions that increase the number of $\mathrm{Bi}$-In neighbors at the surface. These are the first measurements in which resonant $\mathrm{X}$-ray scattering has been used to quantify compositional changes induced at a liquid alloy surface.
\end{abstract}

DOI: 10.1103/PhysRevLett.86.1538

Current treatments of the thermodynamics of surface phenomena in solutions rely heavily on the original works by Gibbs in 1878, and one of the most familiar corollaries is the Gibbs adsorption rule. In its simplest invocation, the Gibbs rule states that, in a binary liquid, the species having the lower surface tension will segregate preferentially at the surface. This apparent simplicity is deceptive: a survey of the literature reveals a hundred years' debate over the application of the Gibbs adsorption rule [1], not to mention its extension to multicomponent systems [2] and crystalline surfaces [3], and its connection to atomistic models [4].

Experimental investigations of the validity of the Gibbs rule encompass measurements of adsorption isotherms [5], surface tension [6], and surface composition [7] in a variety of systems. Unfortunately, many of the liquids studied are too complicated for the simplest formulations of the Gibbs rule. Liquid metal alloys are in many ways ideal for such studies. Miscible alloys exist which behave as ideal liquids, while in other systems strongly attractive or repulsive heteroatomic interactions can be studied. Perhaps an even more important advantage of liquid metals is that the compositionally inhomogeneous region at the surface is known in some cases to be confined to an atomic layer. This is commonly assumed in calculations of Gibbs adsorption that take a model of a physical surface as their starting point.

For example, $\mathrm{x}$-ray reflectivity, ion scattering, and Auger electron spectroscopy measurements of liquid $\mathrm{Ga}_{84} \mathrm{In}_{16}$ found a $94 \%$ In surface monolayer, as expected given this alloy's positive heat of mixing $[8,9]$. Subsequent layers have the bulk composition. Similar studies of dilute liquid $\mathrm{Bi}-\mathrm{Ga}(<0.2$ at. \% Bi) likewise found surface segregation of a pure Bi monolayer [10]. Even when the repulsive interactions between $\mathrm{Ga}$ and $\mathrm{Bi}$ cause more $\mathrm{Bi}$-rich alloys to undergo additional phase separation above $220{ }^{\circ} \mathrm{C}$, where a 65- $\AA$-thick inhomogeneous Bi-rich region forms, the pure Bi surface monolayer persists $[11,12]$. In Ga-Bi, then, re-
PACS numbers: 61.25.-f, 61.10.-i, 68.03.-g

pulsive heteroatomic interactions substantially change the surface composition profile, but do not defeat the Gibbs adsorption.

The effect of attractive heteroatomic interactions remains an open question. In alloys such as Bi-In, attractive forces between the two species produce a number of compositionally ordered phases in the bulk solid. It is therefore conceivable that $\mathrm{Bi}$-In pairing may exist at the liquid surface, and compete with surface segregation. Our recent temperature dependent $\mathrm{x}$-ray reflectivity measurements of liquid Bi-In alloys having 22, 33, and 50 at. \% Bi revealed structural features not found in elemental metals or in the Ga alloys discussed above [13]. As we will show, those data were suggestive of $\mathrm{Bi}$-In pair formation along the surface-normal direction. However, since the technique did not measure the Bi surface concentration directly, other interpretations of the data were also possible.

A complete characterization of surface composition requires both elemental specificity and ̊̊-scale structural resolution along the surface-normal direction, which is difficult to achieve experimentally. Auger electron spectroscopy, which satisfies the first of these requirements, is hampered by contributions from the bulk liquid [7]. X-ray reflectivity by contrast is a surface-sensitive probe. In the kinematic limit [14] the reflected intensity, measured as a function of momentum transfer $q_{z}$ normal to the surface, is proportional to the Fresnel reflectivity $R_{F}$ of a homogeneous surface [15]:

$$
R\left(q_{z}\right)=R_{F}\left|\left(1 / \rho_{\infty}\right) \int_{-\infty}^{\infty}\left(\partial \rho_{\text {eff }} / \partial z\right) \exp \left(i q_{z} z\right) d z\right|^{2} .
$$

Here $\rho_{\text {eff }}$ represents an effective electron scattering amplitude, which combines the electron density profile with the scattering form factor, and $\rho_{\infty}$ is the density of the bulk. The electron density variations that produce modulations in the reflectivity may result from changes in either the composition or the mass density. Thus, inference of surface 
composition from the measured reflectivity is sometimes ambiguous.

This disadvantage can be overcome with the application of resonant x-ray scattering. The effective electron density of a scattering atom depends on the scattering form factor $f(q)+f^{\prime}(q, E) \approx Z+f^{\prime}(E)$. When the x-ray energy is tuned to an absorption edge of a scattering atom, the magnitude of $f^{\prime}$ becomes appreciable, producing changes in contrast between unlike atoms [16]. With one exception [17], resonant x-ray scattering measurements reported in the past have been confined to studies of solids and bulk liquids, due to the difficulty of the experiment. This Letter is the first to find compositional changes induced at a liquid surface.

The molten $\mathrm{Bi}_{22} \mathrm{In}_{78}$ sample was maintained at $T=$ $101{ }^{\circ} \mathrm{C}$ and $P=5 \times 10^{-10}$ Torr, within an ultrahigh vacuum chamber, and periodically sputter cleaned with $\mathrm{Ar}^{+}$ ions. Reflectivity measurements were performed at beam line X25 at the National Synchrotron Light Source. The spectrometer has been described previously [18], except that here a double $\mathrm{Si}(111)$ crystal monochromator was used to provide an energy resolution of $9 \mathrm{eV}$. For this Letter, we compare reflectivity measured at $12.5 \mathrm{keV}$ to measurements made at the Bi $L_{\text {III }}$ edge at $13.421 \mathrm{keV}$. The energy was calibrated by transmission through a Bi foil, shown in Fig. 1(a). At the inflection point indicated by the arrow, $f_{\mathrm{Bi}}^{\prime}$ has its largest magnitude of -24.7 electrons. Uncertainties in $f_{\mathrm{Bi}}^{\prime}$ may arise from inaccuracies in the calculation, incorrect establishment of the incident energy, and the energy resolution. To account for these possibilities, the analysis was performed for deviations in $f_{\mathrm{Bi}}^{\prime}$ of about $20 \%$ (i.e., $f_{\mathrm{Bi}}^{\prime}=-19.7$ and -29.7 electrons). The results were incorporated into the error ranges tabulated below.

Reflectivity data at both energies are shown in Fig. 1(b) (symbols). These results were found to be both repro-
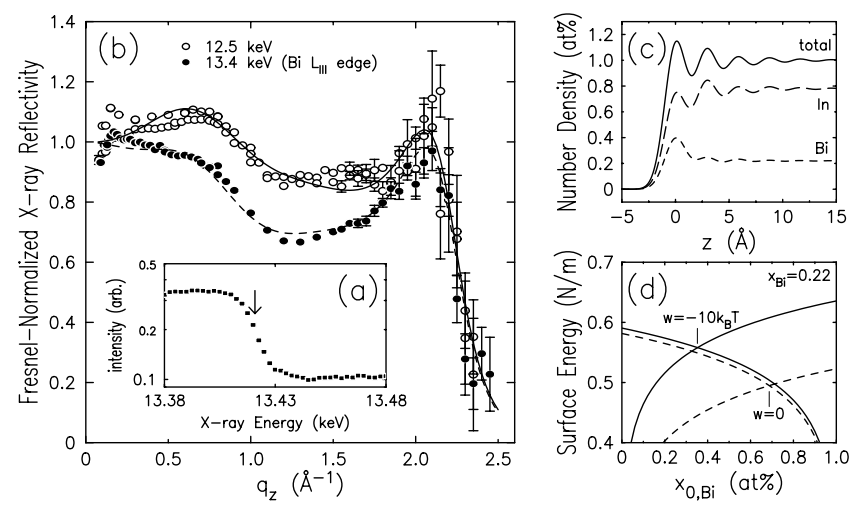

FIG. 1. (a) Energy scan in transmission through $\mathrm{Bi}$ foil. (b) Fresnel-normalized $x$-ray reflectivity of $\mathrm{Bi}_{22} \mathrm{In}_{78} \quad[(\mathrm{O})$ $12.5 \mathrm{keV}$ (two independent measurements), (○) $13.4 \mathrm{keV},(-)$ $12.5 \mathrm{keV}$ fit, and (- - -) $13.4 \mathrm{keV}$ fit]. (c) Best fit real space number density profile relative to bulk atomic percent $[(-)$ Total number density, (- -) In density, and (- - -) Bi density]. (d) Surface energy as a function of surface Bi concentration $x_{0, \mathrm{Bi}}$ for the bulk concentration $x_{\mathrm{Bi}}=0.22$, according to Eq. (5) $\left[(--) w=0\right.$ and $\left.(-) w=-10 k_{B} T\right]$. ducible in energy and stable over time by measuring two $12 \mathrm{keV}$ data sets prior to and following the $13.4 \mathrm{keV}$ measurements. The two $12 \mathrm{keV}$ data sets are shown together as open circles in Fig. 1(b). The interference peak at $q_{z}=2.1 \AA^{-1}$ is due to stratification of the atoms in planes parallel to the surface, a well-established feature of liquid metals [19]. For $q_{z}<1.8 \AA^{-1}$, the data exhibit a modulation indicative of a structural periodicity roughly twice that of the surface layering. This feature is consistent with Bi-In dimers oriented along the surface normal, which could also give rise to alternating $\mathrm{Bi}$ and In layers (bilayers) at the surface. We also find that the low- $q_{z}$ reflectivity is strongly decreased when measured at the $\mathrm{Bi}$ $L_{\text {III }}$ edge. The reflectivity decrease itself varies smoothly with $q_{z}$ and does not exhibit a bilayer-type modulation, instead suggesting that the surface $\mathrm{Bi}$ concentration is larger than that of the bulk.

To investigate these possibilities, we calculate the reflectivity of a model density profile according to Eq. (1), which is refined simultaneously against the data taken at both energies. Resonant effects are included by combining the model structure and the scattering amplitude into an effective electron density profile $\rho_{\text {eff }}(z)$. The energy dependence enters the analysis through the Bi concentration defined in $\rho_{\text {eff }}(z)$, and also through the Fresnel reflectivity $R_{F}$, which is a function of the energy dependent mass absorption coefficient $\mu^{-1}$ and the effective bulk electron density $\rho_{\infty} \propto\left(Z-f^{\prime}\right)$ that defines the critical angle $q_{c}$. Table I shows the values of these quantities used in our models. Reflectivity data acquired at each energy were normalized to the appropriate energy dependent Fresnel function.

Following past practice [18], our model incorporates layers of atoms having a Gaussian distribution of displacements from idealized positions $n d$ along the surfacenormal direction:

$$
\rho_{\mathrm{eff}}(z)=\rho_{\infty} \sum_{n=0}^{\infty} F_{n} \frac{d}{\sigma_{n} \sqrt{2 \pi}} \exp \left[-(z-n d)^{2} / \sigma_{n}^{2}\right] .
$$

The roughness $\sigma_{n}$ arises from both static and dynamic contributions:

$$
\sigma_{n}^{2}=n \bar{\sigma}^{2}+\sigma_{0}^{2}+\frac{k_{B} T}{2 \pi \gamma} \ln \left(\frac{q_{\mathrm{max}}}{q_{\mathrm{res}}}\right) .
$$

Here $\bar{\sigma}$ and $\sigma_{0}$ are related to the surface layering coherence length and the amplitude of density oscillations at the surface. The last term accounts for height fluctuations produced by capillary waves, and depends on the temperature $T=101{ }^{\circ} \mathrm{C}$, the surface tension $\gamma=0.50 \mathrm{~N} / \mathrm{m}$ [20], and wave-vector cutoffs $q_{\max }=0.99 \AA^{-1}$ and $q_{\text {res }} \sim$ $0.024 \AA^{-1}$ (a slowly varying function of $q_{z}$ ), as detailed elsewhere [18].

The scattering amplitude of each layer depends on the form factor and the effective electron density in each layer relative to the bulk, dependent on energy and $\mathrm{Bi}$ 
TABLE I. Parameters used to calculate energy dependent x-ray reflectivity.

\begin{tabular}{ccccccccc}
\hline \hline & $\begin{array}{c}\text { Energy } \\
(\mathrm{keV})\end{array}$ & $\begin{array}{c}\mu / \rho_{m}{ }^{\mathrm{a}} \\
\left(\mathrm{cm}^{2} / \mathrm{g}\right)\end{array}$ & $\begin{array}{c}\rho_{m}{ }^{\mathrm{b}} \\
\left(\mathrm{g} / \mathrm{cm}^{3}\right)\end{array}$ & $\begin{array}{c}\mu^{-1} \\
(\mathrm{~cm})\end{array}$ & $f^{\prime \mathrm{c}}$ & $\left(Z-f^{\prime}\right)$ & $\begin{array}{c}\rho_{\infty} \\
\left(e^{-} / \AA^{3}\right)\end{array}$ & $\begin{array}{c}q_{c} \\
\left(\AA^{-1}\right)\end{array}$ \\
\hline \multirow{2}{*}{$\mathrm{In}$} & 12.5 & 74.1 & 7.0 & 0.00193 & -0.2 & 48.8 & 1.788 & $\cdots$ \\
& 13.4 & 60.5 & & 0.00236 & & & & $\cdots$ \\
$\mathrm{Bi}$ & 12.5 & 75.2 & 10.0 & 0.00133 & -7.3 & 75.7 & 2.180 & $\ldots$ \\
& 13.4 & 155.0 & & 0.000645 & -24.7 & 58.3 & 1.679 & $\cdots$ \\
$\mathrm{Bi}_{22} \mathrm{In}_{78}$ & 12.5 & 74.5 & 7.66 & 0.00175 & $\cdots$ & $\cdots$ & 1.874 & 0.05154 \\
& 13.4 & 92.6 & & 0.00141 & $\cdots$ & $\cdots$ & 1.764 & 0.05000 \\
\hline \hline
\end{tabular}

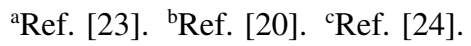

concentration:

$$
F_{n}=w_{n} \times \frac{x_{n, \mathrm{Bi}}\left[f_{\mathrm{Bi}}\left(q_{z}\right)+f_{\mathrm{Bi}}^{\prime}(E)\right]+\left(1-x_{n, \mathrm{Bi}}\right)\left[f_{\mathrm{In}}\left(q_{z}\right)+f_{\mathrm{In}}^{\prime}\right]}{x_{\mathrm{Bi}}\left[Z_{\mathrm{Bi}}+f_{\mathrm{Bi}}^{\prime}(E)\right]+\left(1-x_{\mathrm{Bi}}\right)\left[Z_{\mathrm{In}}+f_{\mathrm{In}}^{\prime}\right]} \times \frac{x_{n, \mathrm{Bi}} \rho_{\infty, \mathrm{Bi}}+\left(1-x_{n, \mathrm{Bi}}\right) \rho_{\infty, \mathrm{In}}}{\rho_{\infty, \text { bulk }}} .
$$

For the first few layers $(n=0,1,2)$, the weight $w_{n}$ may differ from unity and the Bi fraction $x_{n, \mathrm{Bi}}$ can vary from the bulk value $x_{\mathrm{Bi}}=0.22$.

We now describe the ingredients that are required to fit the data with this model. The interference peak at $q_{z}=2.1 \AA^{-1}$ can be reproduced by a simple layered profile in which $x_{n, \mathrm{Bi}}=x_{\mathrm{Bi}}$ for all $n$. The Bi and In number densities for such a model are shown in Fig. 2(a)i, along with their sum, the total atomic fraction relative to the bulk. The corresponding reflectivity curves calculated for both x-ray energies are compared to the experimental data in Fig. 2(b)i. Since the Bi concentration is uniform, the energy dependence is so slight that the curves overlap almost completely on the scale of the figure. Turning again to the data, the reduced intensity in the region $q_{z}<1.8 \AA^{-1}$ when measured at the Bi $L_{\text {III }}$ edge indicates a reduction in the scattering amplitude at the surface. This implies that the Bi concentration is enhanced there.
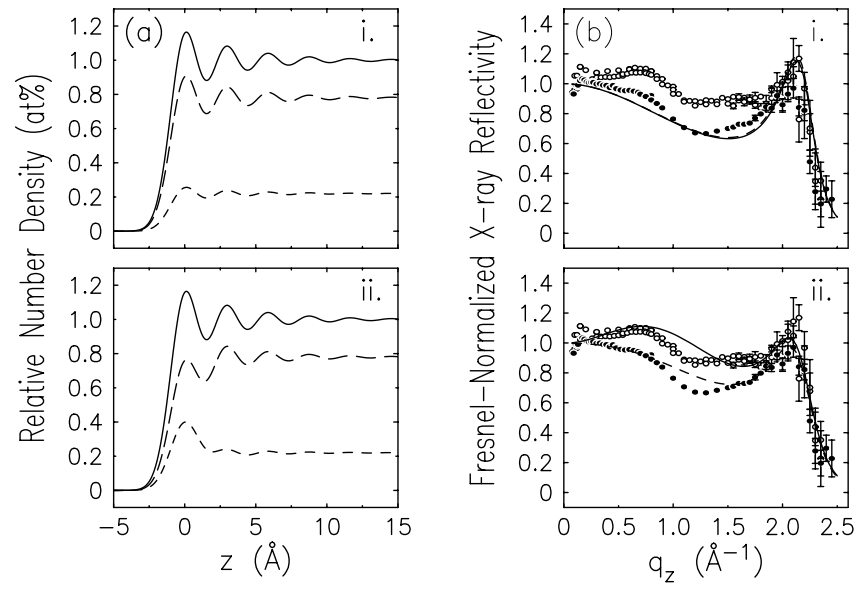

FIG. 2. (a) Model surface-normal number density profiles, relative to bulk atomic percent $[(-)$ Total number density, (- -) In density, and (- - -) Bi density]. (b) Calculated Fresnel-normalized reflectivity curves $[(-) 12.5 \mathrm{keV}$ and (- - ) $13.4 \mathrm{keV}$. (i) Surface layering with uniform composition $\left(x_{\mathrm{Bi}}=0.22\right)$. (ii) Surface layering, with Bi enhancement in the first atomic layer $\left(x_{0, \mathrm{Bi}}=0.35\right)$.
Increasing the $\mathrm{Bi}$ fraction from 22 at. $\%$ to $\approx 35$ at. $\%$ in the first layer $(n=0)$ produces an appropriate energy dependence [Figs. 2(a)ii and 2(b)ii].

Although at this point the frequency of the low- $q_{z}$ modulation is not well described, the fit is considerably improved by allowing the $\mathrm{Bi}$ fraction and total number densities to vary for the first three surface layers $(n=0,1,2)$. We find that the Bi fraction for $n=1,2$ is essentially equal to the bulk value of 22 at. \%, while the total number densities for $n=0,1,2$ have values of $0.98,1.01$, and 0.98, respectively [Figs. 1(b) and 1(c)]. Thus, the detailed shape of the low- $q_{z}$ modulation is modeled by a very slight density wave of about $2 \%$ affecting the amplitudes of the first few surface layers. Increasing the number of model parameters to allow for shifts in positions and widths of the surface layers resulted in marginally better fits, but at the expense of high frequency Fourier components appearing as small wiggles in the calculated reflectivity. Variations in these extra fit parameters are extremely slight, and we doubt whether they have any physical basis. Parameters for all models are shown in Table II.

This analysis demonstrates that, to model the essential features of our data, there is no need to invoke longrange compositional ordering on a second length scale in the surface-normal direction, which we had suggested based on the previous nonresonant reflectivity measurements $[11,13]$. Still, we thought it important to investigate additional, specific models based on Bi-In pairs oriented along the surface normal. To test for alternating Bi-rich and In-rich layers, we attempted fits in which we forced the $\mathrm{Bi}$ and In compositions to be substantially different from the results shown above. We also described Bi-In pairing by allowing the positions, but not the densities, of the surface layers to vary. None of these profiles successfully described the data.

Our principal finding is the Bi enrichment of 35 at. \% in the surface layer, compared to the bulk value of 22 at. $\%$. This is considerably less Bi than would be expected in 
TABLE II. Fit parameters for model profiles, identified by the figure in which they appear. The length scales $d, \bar{\sigma}$, and $\sigma_{0}$ are in units of $\AA$.

\begin{tabular}{|c|c|c|c|c|c|c|c|c|c|}
\hline Fit & $d$ & $\bar{\sigma}$ & $\sigma_{0}$ & $w_{0}$ & $x_{0, \mathrm{Bi}}$ & $w_{1}$ & $x_{1, \mathrm{Bi}}$ & $w_{2}$ & $x_{2, \mathrm{Bi}}$ \\
\hline Fig. 1 & $2.81(2)$ & $0.54(4)$ & $0.64(2)$ & $0.98(2)$ & $0.35(4)$ & $1.01(1)$ & $0.22(2)$ & $0.98(1)$ & $0.23(2)$ \\
\hline Figs. 2(a)i-2(b)i & 2.81 & 0.48 & 0.64 & 1.0 & 0.22 & 1.0 & 0.22 & 1.0 & 0.22 \\
\hline Figs. 2(a)ii-2(b)ii & 2.81 & 0.54 & 0.64 & 1.0 & 0.35 & 1.0 & 0.22 & 1.0 & 0.22 \\
\hline
\end{tabular}

the absence of attractive Bi-In interactions, which can be estimated from the surface free energy requirement [21]:

$$
\gamma_{\mathrm{In}}+\frac{k_{B} T}{a} \ln \left(\frac{1-x_{0, \mathrm{Bi}}}{1-x_{\mathrm{Bi}}}\right)-\frac{1}{4}\left(x_{\mathrm{Bi}}\right)^{2} \frac{w}{a}=\gamma_{\mathrm{Bi}}+\frac{k_{B} T}{a} \ln \left(\frac{x_{0, \mathrm{Bi}}}{x_{\mathrm{Bi}}}\right)-\frac{1}{4}\left(1-x_{\mathrm{Bi}}\right)^{2} \frac{w}{a} .
$$

The quantity $w$ is the excess interaction energy of Bi-In pairs over the average of the $\mathrm{Bi}-\mathrm{Bi}$ and In-In interaction energies; for an ideal mixture, $w=0$. This analysis assumes that the inhomogeneous region is confined to a single atomic layer, the atoms are close-packed and take up an area $a$, and $w$ is small. Extrapolating the measured surface tensions to $100{ }^{\circ} \mathrm{C}[20], \gamma_{\text {In }}=0.56 \mathrm{~N} / \mathrm{m}$ and $\gamma_{\mathrm{Bi}}=0.41 \mathrm{~N} / \mathrm{m}$. By using the Bi atomic size, $a=$ $\pi(3.34 / 2)^{2} \AA^{2}$ (for In, the atomic diameter is $3.14 \AA$ ) [22], and the bulk composition $x_{\mathrm{Bi}}=0.22$. The equilibrium surface composition $x_{0, \mathrm{Bi}}$ is found from the intersection of plots of both sides of Eq. (5). For $w=0$, this analysis predicts a surface segregation of 69 at. \% Bi [Fig. 1(d), dashed lines]. To reproduce our experimental finding that $x_{0, \mathrm{Bi}}=35$ at. $\%, w$ must be negative, with a magnitude of $\sim 10 k_{B} T$ [Fig. 1(d), solid lines]. Although this large value of $w$ is most likely outside the range of validity of Eq. (5), the analysis certainly illustrates the qualitative effect of attractive heteroatomic interactions on the surface composition. In this Bi-In alloy, pairing does in fact defeat Gibbs adsorption in the sense that the surface energy is optimized not by segregating a large fraction of $\mathrm{Bi}$, but by forming larger numbers of $\mathrm{Bi}$-In neighbors in the surface layer. Exactly how this balance plays out in Bi-In alloys with the stoichiometric bulk compositions BiIn and $\mathrm{BiIn}_{2}$ remains to be seen.

This work is supported by U.S. DOE Grant No. DEFG02-88-ER45379 and No. DE-AC02-98CH10886, and by the U.S.-Israel Binational Science Foundation, Jerusalem. P. H. acknowledges support from the Deutsche Forschungsgemeinschaft. Use of the CMC-CAT liquid surface spectrometer was supported under U.S. DOE Contract No. W-31-109-Eng-38 and the NSF-DMR.

*Author to whom correspondence should be addressed. Electronic address: dimasi@bnl.gov

${ }^{\dagger}$ Present address: Department of Chemistry, University of Florida, Gainesville, Florida 32611.

[1] J. W. Cahn and J.E. Hilliard, J. Chem. Phys. 28, 258 (1958); D. W. G. White, Metall. Rev. 124, 73 (1968); R. A. Alberty, Langmuir 11, 3598 (1995).

[2] B. Widom, Physica (Amsterdam) 95A, 1 (1979).

[3] A. I. Rusanov, Surf. Sci. Rep. 23, 173 (1996); V. V. Bakovets, Phys. Status Solidi (b) 205, 507 (1998).
[4] R. Speiser et al., Scr. Metall. 21, 687 (1987).

[5] A. W. Adamson, Physical Chemistry of Surfaces (Wiley, New York, 1960).

[6] A. B. Bhatia and N. H. March, J. Chem. Phys. 68, 4651 (1978); Y. Oguchi et al., Phys. Chem. Liq. 10, 315 (1981).

[7] S. Hardy and J. Fine, in Materials Processing in the Reduced Gravity Environment of Space, edited by G. E. Rindone (Elsevier, The Netherlands, 1982), p. 503.

[8] M. J. Regan et al., Phys. Rev. B 55, 15874 (1997).

[9] M.F. Dumke et al., Surf. Sci. 124, 407 (1983).

[10] N. Lei et al., J. Phys. Chem. 104, 4802 (1996); 105, 9615 (1996).

[11] E. DiMasi and H. Tostmann, Synchrotron Radiation News 12, 41 (1999).

[12] H. Tostmann et al., Phys. Rev. Lett. 84, 4385 (2000).

[13] E. DiMasi et al., J. Phys. Condens. Matter 12, A209 (2000); E. DiMasi et al. (to be published).

[14] The kinematic limit, or Born approximation, is valid where $R\left(q_{z}\right) \ll 1$. In this paper, data are fit only for $q_{z}>0.2$, where $R\left(q_{z}\right)<3 \times 10^{-4}$, and the structure is determined principally by data with $q_{z}>0.4$, where $R\left(q_{z}\right)$ is in the range $10^{-5}-10^{-9}$.

[15] A. Braslau et al., Phys. Rev. A 38, 2457 (1988).

[16] Resonant Anomalous X-ray Scattering: Theory and Applications, edited by G. Materlik, C. J. Sparks, and K. Fischer (North-Holland, Amsterdam, 1994), p. 47.

[17] E. DiMasi et al., Mater. Res. Soc. Symp. Proc. 590, 183 (2000).

[18] H. Tostmann et al., Phys. Rev. B 59, 783 (1999).

[19] M.P. D'Evelyn and S. A. Rice, Phys. Rev. Lett. 47, 1844 (1981); S. A. Rice, J. Non-Cryst. Solids 205-207, 755 (1996), and references therein.

[20] This estimate for $\gamma$ is an average of the In and $\mathrm{Bi}$ surface tensions extrapolated to $100^{\circ}$ [data from the $C R C$ Handbook of Chemistry and Physics, edited by R. C. Weast (CRC Press, Boca Raton, FL, 1985)]. Inaccuracies in $\gamma$ are compensated by $\sigma_{0}$ in Eq. (3), and do not otherwise affect the results.

[21] E. A. Guggenheim, Mixtures (Clarendon Press, London, 1952), Chap. IX.

[22] T. Iida and R. I. L. Guthrie, The Physical Properties of Liquid Metals (Clarendon Press, Oxford, 1993), p. 35.

[23] International Tables for X-Ray Crystallography, edited by C. H. MacGillavry and G. D. Rieck (Kynoch Press, Birmingham, England, 1962), Vol. III.

[24] B. L. Henke, E. M. Gullikson, and J.C. Davis, At. Data Nucl. Data Tables 54, 181 (1993). 